\section{Should Patients Continue to Receive Statins Once They Reach 80 Years of Age?}

\section{THE "PRO" SIDE}

Statins are not evil, they are just misunderstood. I have observed other health care professionals (particularly pharmacists), upon discovering that an elderly patient is taking a statin, react with incredulous disgust. "What are they doing to this poor person?" they say, as if the prescriber were contravening the Declaration of Helsinki. Some clinicians make flippant decisions to stop statins simply because patients are in their 80 s or beyond. However, despite their perceived negative reputation, statins are conspicuously absent from the American Geriatric Society's 2015 updated Beers Criteria for potentially inappropriate medication use in older adults. ${ }^{1}$ That list does include other ubiquitous drugs, such as $\alpha_{1}$ blockers, $\alpha_{2}$ agonists, amiodarone, and digoxin, but we are not debating the appropriateness of using these drugs in older patients. Is that because we are so enlightened in practice that we no longer use these agents in older patients (a proposition that I would fervently dispute), or do statins suffer ill repute for some reason when it comes to persons of advanced age? I would argue the latter.

To be explicit, statin discontinuation should never be based solely on age, like a life insurance policy that somehow expires on one's 80th birthday. All therapeutic decisions, related to statins or otherwise, should be based on functional age, as opposed to chronological age. When considering starting or stopping statin therapy in an older patient, the clinician must consider frailty and/or functionality, life expectancy, comorbidities, polypharmacy, and the patient's goals of therapy.

One common argument for statin discontinuation in elderly patients is based on life expectancy. Although some patients may not live long enough to benefit, it is not as if the Kaplan-Meier curves derived from statin studies will sudden deviate after 4 or 5 years of therapy. Statins may lower the risk of cardiovascular events in fewer years than some assume. For example, the JUPITER trial was discontinued early because of benefit after a median follow-up of only 1.9 years. ${ }^{2}$ Acknowledging that the interquartile age range in that study was 60-71 years, I would argue that these data are relevant to older adults despite the study not being limited to those over 80 years of age. Thus, it is reasonable to consider a statin for patients with life expectancy of at least 2 years.
Another common argument is that elderly people are underrepresented in clinical trials, which is true, just as it is for women and persons of various ethnic backgrounds. But do we always refrain from using a particular therapy in certain individuals just because they are different from the majority of the study population? In practice, we often fail to identify our own hypocrisy in extrapolating from the evidence. We are comfortable recommending other cardiovascular secondary prevention therapy (e.g., acetylsalicylic acid, ß-blockers, angiotensin-converting enzyme inhibitors) for those over 80 years of age, regardless of the deficiency of evidence specific to this population, yet balk at the suggestion of using statins. Furthermore, I would argue that these agents are potentially more harmful than statins in older patients because of the risks of gastrointestinal bleeding, heart block, and hypotension or renal impairment, respectively.

Absence of evidence does not necessarily indicate an absence of benefit. Granted, the contrary is also true (i.e., absence of evidence does not preclude harm), but the data we do have suggest that patients near 80 years of age benefit from statin therapy.

The PROSPER randomized controlled trial (RCT) enrolled 5804 patients between the ages of 70 and 82 years (mean \pm standard deviation $75 \pm 3$ years) with established, or at risk for, cardiovascular disease, who were randomly assigned to receive pravastatin $40 \mathrm{mg}$ daily or placebo. ${ }^{3}$ After a mean follow-up period of 3.2 years, the primary outcome-composite of coronary heart disease death, nonfatal myocardial infarction, and fatal or nonfatal stroke-was reduced by $2.1 \%$ (number needed to treat [NNT] 48). Death from any cause was similar between the groups, with no significant difference in the risk of myalgia.

A meta-analysis of 8 RCTs investigated statin therapy for primary cardiovascular prevention in 24674 patients 65 years of age or older (mean $73 \pm 3$ years). ${ }^{4}$ Over a mean follow-up of 3.5 years, statin therapy reduced myocardial infarction by $1.2 \%$ (NNT 84) and stroke by $0.7 \%$ (NNT 143), with no significant difference in all-cause or cardiovascular death or in the risk of new cancer. Another meta-analysis included 9 RCTs totalling 19569 patients aged 65-82 years with documented coronary heart disease. ${ }^{5}$ Over a mean follow-up period of 4.9 years, statin therapy, relative to placebo, reduced all-cause mortality by 3.2\% (NNT 32 ), coronary heart disease mortality by $2.6 \%$ (NNT 39), nonfatal myocardial infarction by $2.5 \%$ (NNT 40), and stroke 
by $1.8 \%$ (NNT 56). Although the number of patients 80 years of age or older was not reported in these meta-analyses, it seems unsound to simply disregard these results for that age group. Furthermore, preventing a cardiovascular event, such as myocardial infarction, in older patients is not trivial, as it keeps them out of hospital.

The same "paucity of beneficial evidence" argument for statins in those 80 years or older also applies to safety. It is incongruous to dismiss the efficacy of statins in older persons and at the same time empirically discontinue therapy because of potential adverse effects. One supposed effect of particular concern for older adults is cognitive impairment. Multiple systematic reviews and meta-analyses have demonstrated either no association between statins and cognitive impairment ${ }^{6-8}$ or possibly a reduction in incident dementia. ${ }^{9,10}$ Advanced age is, however, a risk factor for statin-associated myopathy, as are female sex, low body mass index, frailty, and renal disease. ${ }^{11}$ Older patients who are receiving statin therapy should be monitored frequently for adverse effects. However, that does not imply that the use of a high-potency statin in an asymptomatic 80-year-old patient is cruel and unusual.

Should patients continue to receive statins once they reach 80 years of age? In the end, this is a specious argument because the decision is not really up to us. We are expected to support our older patients in making informed decisions based on the evidence, and to avoid the temptation to devalue their input by impulsively surmising that statin therapy is contraindicated because of age alone.

\section{References}

1. American Geriatrics Society 2015 Beers Criteria Update Expert Panel. American Geriatrics Society 2015 updated Beers Criteria for potentially inappropriate medication use in older adults. J Am Geriatr Soc. 2015; 63(11):2227-46.

2. Ridker PM, Danielson E, Fonseca FAH, Genest J, Gotto AM Jr, Kastelein JJ, et al.; JUPITER Study Group. Rosuvastatin to prevent vascular events in men and women with elevated C-reactive protein. N Engl J Med. 2008; 359(21):2195-207.

3. Shepherd J, Blauw GJ, Murphy MB, Bollen EL, Buckley BM, Cobbe SM, et al.; PROSPER Study Group. Pravastatin in elderly individuals at risk of vascular disease (PROSPER): a randomised controlled trial. Lancet. 2002; 360(9346):1623-30

4. Savarese G, Gotto AM Jr, Paolillo S, D’Amore C, Losco T, Musella F, et al. Benefits of statins in elderly subjects without established cardiovascular disease: a meta-analysis. J Am Coll Cardiol. 2013;62(22):2090-9.

5. Afilalo J, Duque G, Steele R, Jukema JW, de Craen AJM, Eisenberg MJ. Statins for secondary prevention in elderly patients: a hierarchical Bayesian meta-analysis. J Am Coll Cardiol. 2008;51(1):37-45.

6. Richardson K, Schoen M, French B, Umscheid CA, Mitchell MD, Arnold $\mathrm{SE}$, et al. Statins and cognitive function: a systematic review. Ann Intern Med. 2013;159(10):688-97.

7. Ott BR, Daiello LA, Dahabreh IJ, Springate BA, Bixby K, Murali M, et al. Do statins impair cognition? A systematic review and meta-analysis of randomized controlled trials. J Gen Intern Med. 2015;30(3):348-58.

8. McGuinness B, Craig D, Bullock R, Passmore P. Statins for the prevention of dementia. Cochrane Database Syst Rev. 2016;(1):CD003160.

9. Swiger KJ, Manalac RJ, Blumenthal RS, Blaha MJ, Martin SS. Statins and cognition: a systematic review and meta-analysis of short- and long-term cognitive effects. Mayo Clin Proc. 2013;88(11):1213-21.

10. Macedo AF, Taylor FC, Casas JP, Adler A, Prieto-Merino D, Ebrahim S.
Unintended effects of statins from observational studies in the general population: systematic review and meta-analysis. BMC Med. 2014;12:51.

11. Mancini GB, Baker S, Bergeron J, Fitchett D, Frohlich J, Genest J, et al. Diagnosis, prevention, and management of statin adverse effects and intolerance: proceedings of a Canadian Working Group Consensus Conference. Can J Cardiol. 2011;27(5):635-62.

Arden R Barry, BSC, BSc(Pharm), PharmD, ACPR

Chilliwack General Hospital

Lower Mainland Pharmacy Services

Chilliwack, British Columbia

Arden Barry is also with the Faculty of Pharmaceutical Sciences, The University of British Columbia, Vancouver, British Columbia.

Competing interests: Dr Barry was a primary panel member and coauthor of the 2016 Canadian Cardiovascular Society Guidelines for the Management of Dyslipidemia for the Prevention of Cardiovascular Disease in the Adult. No other competing interests were declared.

\section{THE "CON" SIDE}

Should patients in their 80 s and beyond continue to receive statins? More generally, is any clinician in a position to instruct patients as to what they should or should not take? As clinicians, none of us can direct patients to take a particular medication; rather, each of us has the responsibility to facilitate an informed decision by the patient. In the case of statins, the informed decision must be sensitive to key issues related to statin evidence. Here, we will attempt to outline some, though not all, of these issues.

\section{The Net Effect (Balance of Benefits and Harms) of Statins in Older Patients Has Not Been Determined}

Patients should be made aware that few patients over 80 years of age have been included in statin trials. A recent systematic review of statin trials for primary prevention in older patients found that the average age of participants was 73 years. ${ }^{1}$ Similarly, a systematic review of statin trials for secondary prevention in older patients found that the average was 69 years. $^{2}$ As such, patients over 80 years of age represent only a small subgroup of all patients enrolled in the trials, which leaves uncertainty about the treatment effect of statins in this older age group. ${ }^{3}$ The statin evidence is best generalized to middle-aged, white men. ${ }^{4}$

\section{We Don't Really Know the Impact of Stopping Statins in Adults, Let Alone Older Adults}

Limited and conflicting evidence exists on the impact of stopping statins. ${ }^{5}$ One observational study suggested an increased risk of cardiovascular events after discontinuing statin therapy, ${ }^{6}$ but these results should be interpreted with caution because of the observational study design and the presence of residual confounding. ${ }^{7}$ One small 
randomized trial of patients with limited life expectancy suggested no difference in mortality within 60 days and improved quality of life with stopping versus continuation of statins. ${ }^{8}$ To date, evidence specific to patients over 80 years of age is lacking.

\section{Statin Treatment Decisions Are Typically Based on Low-Density-Lipoprotein Cholesterol}

Statin treatment decisions are often based on levels of lowdensity-lipoprotein (LDL) cholesterol, with elevated levels typically leading to initiation of a statin, an increase in dose, or continuation of statin therapy. This approach is based on the common assumption that elevated LDL cholesterol levels are always associated with increased risk of morbidity and mortality. However, evidence is emerging that this association is reversed in older adults. An analysis of 30 observational studies demonstrated that low LDL cholesterol was associated with increased all-cause mortality (28 studies) or no relationship to this outcome (2 studies). ${ }^{9}$ Similar results have been documented for cardiovascular mortality: in 7 studies, patients with low LDL cholesterol had a higher risk, and 2 studies found no association.' In light of this evidence, starting, continuing, or intensifying statin therapy in older adults on the basis of LDL cholesterol levels may be unwise.

\section{Statins Can Cause Important Harm}

Patients over 80 years of age should be informed of the possible harm associated with statins and the likelihood that they will experience this harm. There is evidence from randomized controlled trials (RCTs) and observational studies in adults that statins can increase the risk of acute kidney injury (number needed to harm [NNH] 450), cataracts ( $\mathrm{NNH} 50$ ), hemorrhagic stroke ( $\mathrm{NNH} 108$ ), muscle pain or damage (NNH 48 for musculoskeletal conditions, NNH 100 for moderate to serious myopathy), and moderate to serious liver dysfunction (NNH 150). In addition, it is estimated that 1 in 50 patients will withdraw from statin therapy because of an adverse event of any type or severity. ${ }^{10}$

\section{Benefits and Likelihood of "Success" May Be Exaggerated, While Harms Are Underestimated}

Published RCTs of statins may exaggerate their beneficial effects and underestimate their harms. ${ }^{11}$ Statin trials generally employ run-in periods, whereby those at risk of harm and those least likely to have beneficial effects have not been included in the randomization. ${ }^{12}$ Finally, blinding was easily compromised in most of the trials because clinicians had access to lipid profiles. ${ }^{13}$

Beyond these concerns, when we use the phrase "should take a statin" we inadvertently imply that the statin will fully eliminate the risk of bad outcomes. Even the most optimistic assessment of evidence for primary prevention with statins in older patients (mean age 73 years) suggests absolute risk reductions of $1.2 \%$ for myocardial infarction (NNT 83) and $0.7 \%$ for stroke (NNT 166) over 3.5 years, with no reduction in mortality. ${ }^{1}$ For secondary prevention with statins in older patients (mean age 69 years), the most optimistic assessment of the evidence suggests an absolute risk reduction of $2.6 \%$ for myocardial infarction (NNT 83), 1.7\% for stroke (NNT 58), and $3.8 \%$ for all-cause mortality (NNT 28) over 3.5 years. ${ }^{2}$ It should be noted that limited information is available on the impact of statins on the risk of disabling stroke, an important goal of therapy in older adults.

What does this mean for individual patients over 80 years of age? To illustrate with a primary prevention example, it means that 82 of 83 patients treated with statins will not experience the reduction in risk of myocardial infarction. This hardly represents an elimination of risk. In addition, there is no way to know whether an individual patient will derive this effect from a statin. It is important that patients understand these concepts in order to set realistic expectations for themselves.

\section{Informed Decision-Making Is Critical, Not Paternalistic Prescribing}

An informed decision is important for anyone considering whether to start or continue statins, because, as we have outlined, there is considerable uncertainty in the evidence. Each patient over 80 years of age will need to consider what matters most and will need all the available information regarding statins before they can decide. $^{14}$

It is important to note that our approach here has been to provide a high-level overview of the available published evidence. A detailed critical appraisal of all published and unpublished information related to the trials of statins in elderly people (e.g., clinical study reports, regulatory documents, trial protocols) is not possible in this context.

References

1. Savarese G, Gotto AM Jr, Paolillo S, D'Amore C, Losco T, Musella F, et al. Benefits of statins in elderly subjects without established cardiovascular disease: a meta-analysis. J Am Coll Cardiol. 2013;62(22):2090-9.

2. Afilalo J, Duque G, Steele R, Jukema JW, de Craen AJ, Eisenberg MJ. Statins for secondary prevention in elderly patients: a hierarchical Bayesian meta-analysis. J Am Coll Cardiol. 2008;51(1):37-45.

3. Sun X, Briel M, Walter SD, Guyatt GH. Is a subgroup effect believable? Updating criteria to evaluate the credibility of subgroup analyses. BMJ. 2010;340:c117.

4. Therapeutics Initiative. High dose versus standard dose statins in stable coronary heart disease. Ther Lett. 2012;87:1-2. Available from: www.ti.ubc.ca/2013/01/02/high-dose-versus-standard-dose-statins-in-stablecoronary-heart-disease/

5. Marrs JC, Kostoff MD. Discontinuation of statins: what are the risks? Curr Atheroscler Rep. 2016;18(7):41.

6. Nielsen SF, Nordestgaard BG. Negative statin-related news stories decrease statin persistence and increase myocardial infarction and cardiovascular mortality: a nationwide prospective cohort study. Eur Heart J. 2016;37(11): 908-16.

7. Filion KB, Schneider-Lidner V, Karp I, Lévesque LE, Brophy JM, Suissa S. Continuation of statin treatment and mortality: a note of caution on excessive benefits. Arch Intern Med. 2009;169(11):1080 (author reply 1080-1).

8. Kutner JS, Blatchford PJ, Taylor DH Jr, Ritchie CS, Bull JH, Fairclough DL, et al. Safety and benefit of discontinuing statin therapy in the setting of advanced, life-limiting illness: a randomized clinical trial. JAMA Intern Med. 2015;175(5):691-700. 
9. Ravnskov U, Diamond DM, Hama R, Hamazaki T, Hammarskjöld B, Hynes $\mathrm{N}$, et al. Lack of an association or an inverse association between low-density-lipoprotein cholesterol and mortality in the elderly: a systematic review. BMJ Open. 2016;6(6):e010401.

10. Therapeutics Initiative. Statins: proven and associated harms. Ther Lett. 2016;89:1-2. Available from: www.ti.ubc.ca/2014/05/28/statins-proven-andassociated-harms/

11. Lundh A, Lexchin J, Mintzes B, Schroll JB, Bero L. Industry sponsorship and research outcome. Cochrane Database Syst Rev. 2017;2:MR000033.

12. Pablos-Méndez A, Barr G, Shea S. Run-in periods in randomized trials: implications for the application of results in clinical practice. JAMA. 1998; 279(3):222-5

13. Savović J, Jones HE, Altman DG, Harris RJ, Jüni P, Pildal J, et al. Influence of reported study design characteristics on intervention effect estimates from randomized, controlled trials. Ann Intern Med. 2012;157(6):429-38.

14. Elwyn G, Frosch D, Thomson R, Joseph-Williams N, Lloyd A, Kinnersley P, et al. Shared decision making: a model for clinical practice. J Gen Intern Med. 2012;27(10):1361-7.
Aaron M Tejani, BSc(Pharm), PharmD

Cait O'Sullivan, PharmD, BScPh, BA

Therapeutics Initiative

The University of British Columbia

Vancouver, British Columbia

Competing interests: For activities not directly related to the topic of this article, Aaron Tejani has received a grant from the College of Pharmacists of British Columbia and payment for lectures on a variety of topics from The University of British Columbia, Vancouver Island Health Authority Pharmacy Services, and various professional societies and organizations. No other competing interests were declared.

\section{CISADL 2016}

\section{The 2016 Canadian Investigational \& Special Access Drug List is now on sale!}

Please use the CSHP Products \& Publications Order Form found online at CSHP.ca to place your

order: http://www.cshp.ca/productsServices/otherPublications/canadianInvestigational e.asp

The Canadian Investigational \& Special Access Drug List (CISADL) is a compilation of drugs that are currently not marketed in Canada, and may have investigational or special access status with Health Canada. This list is not endorsed by Health Canada's Special Access Program. For precise information about the regulatory status of a drug, contact the Special Access Program directly. Entries to the list are contributed to by several drug information centres across Canada and by Canadian drug manufacturers. In cases where non-marketed drugs do not appear on the list, foreign references should be consulted. The content of the list includes generic name and strength/concentration, dosage form, pharmacology/therapeutic use, trade and code name, source (manufacturer/distributor), and comments. Available in English only. 